\title{
INTERPOLATION SERIES
}

BY

\section{R. CREIGHTON BUCK}

1. Introduction. There is an extensive literature devoted to the study of the expansion of analytic functions into the interpolation series defined by a sequence $\left\{T_{n}\right\}$ of linear functionals. Some of these deal with specific series, and the problem is to determine the largest class of functions for which a given series is convergent $[6 ; 11 ; 15 ; 21 ; 23]\left({ }^{1}\right)$. Others treat a more or less general class of interpolation series; the results obtainable here are seldom as sharp as those obtained for a single series $[1 ; 5 ; 10 ; 19 ; 20 ; 24]$. A closely related problem is that of determining uniqueness classes. If $C$ is a class of functions, $\left\{T_{n}\right\}$ is said to be total over $C$ if $f \in C$ and $T_{n}(f)=0$ for all $n$ imply that $f=0 ; C$ is also said to be a uniqueness class for $\left\{T_{n}\right\}$, since if $T_{n}(f)=T_{n}(g)$ for all $n$, and for $f$ and $g$ in $C$, then $f=g$. If $C$ is an expansion class for $\left\{T_{n}\right\}$ then it is also a uniqueness class. This remark has been used to obtain uniqueness classes for certain special sequences of functionals.

In the present paper, certain aspects of the convergence and summability of interpolation series will be discussed, following a method outlined in [4]. $\S \S 2$ and 3 are devoted to the general treatment; succeeding sections take up in more detail the results which follow for special series. These contain and extend many of the known results. In addition, the theorems dealing with Mittag-Leffler summability are applied in $\$ 10$ to obtain uniqueness theorems which in particular include all those of Gelfond [10]. Some of these results have been announced in a previous paper [4].

2. Integral formulae. We are concerned with the class $K$ of entire functions of exponential type. If $f \in K$, then the growth function of $f$ is defined by

$$
h(\theta, f)=\lim \sup r^{-1} \log \left|f\left(r e^{i \theta}\right)\right| .
$$

If $H$ is any real function with period $2 \pi$, then $K[H(\theta)]$ is the class of all $f$ in $K$ such that $h(\theta, f) \leqq H(\theta)$ for all $\theta$; infinite values of $H$ are admissible. Thus, $K[A]$ is the class of entire functions of at most order 1 and type $A$. If $\psi$ is a positive real function, then $K[\psi(r), H(\theta)]$ will be used for the class of all $f$ in $K$ such that the inequality

$$
\left|f\left(r e^{i \theta}\right)\right| \leqq \psi(r) \exp r H(\theta)
$$

holds for all $\theta$, and all sufficiently large $r$. The class $K[H(\theta)]$ is the intersection

Presented to the Society, November 2, 1946; received by the editors July 14, 1947.

(1) Number in brackets refer to the bibliography at the end of the paper. 
of the classes $K\left[e^{\lambda r}, H(\theta)\right]$ for all $\lambda>0$. As in previous papers, $K(a, c)$ will be used in place of $K[H(\theta)]$ when $H$ is chosen as the special function which takes the value $c$ at $\pm \pi / 2$, and the value $a$ at 0 and $\pi$. (This class arises whenever $f$ is studied from its behavior on the real axis.)

If $G$ is a closed set of the plane, a supporting function for it is defined by

$$
k(\theta, G)=\sup _{w \in G} \Re\left(w e^{i \theta}\right)
$$

where $w=\rho e^{i \phi}$. (This differs from the usual definition in the sign of $\theta$.) The function $k(\theta, G)$ is continuous in $\theta$, has period $2 \pi$, has left and right derivatives everywhere, and is unchanged if $G$ is replaced by its convex hull. If $G_{1}$ and $G_{2}$ are subsets of the plane then $G_{1} \cdot G_{2}$ is the set of all points of the form $z_{1} z_{2}$ where $z_{1} \in G_{1}, z_{2} \in G_{2}\left({ }^{2}\right) . G$ is a star set (with respect to the origin) if $\lambda z \in G$ for all $\lambda, 0 \leqq \lambda \leqq 1$, whenever $z \in G$. The set $\left(E^{\prime} \cdot G^{\prime}\right)^{\prime}$ will play a special role in succeeding sections, and will be referred to as the $E$-star of $G$. If $E$ is chosen as the whole plane with the interval $[1,+\infty]$ of the reals deleted, then the $E$-star of $G$ becomes simply the star of $G$-that is, the maximal star set contained in $G$.

A classical result of Polya relates the growth function of $f$ to the supporting function of a certain convex set. Let $f \in K$ and suppose that $f(z)=\sum_{0}^{\infty} a_{n} z^{n} / n$ ! ; let $\phi(w)$ be the function regular at infinity having the development $\sum_{0}^{\infty} a_{n} / w^{n+1} . \phi$ is usually called the Borel transform of $f$. Let $D(f)$ be the closed convex hull of the set of singularities of $\phi$, a bounded set. Then, the fundamental theorem of Pólya may be stated as follows [16, p. 585] :

If $f \in K$, then $h(\theta, f)=k(\theta, D(f))$ for all $\theta$. Moreover, if $\Gamma$ is a simple contour enclosing $D(f)$, then for all $z$,

$$
f(z)=\frac{1}{2 \pi i} \int_{\Gamma} e^{z w} \phi(w) d w .
$$

Recently, S. Schmidli [20] has obtained a stronger form of this representation which may be stated in the following form $\left(^{(3)}\right.$ :

If $f \in K[\psi(r), h(\theta, f)]$ where

$$
\int^{\infty} \psi(r) d r<\infty
$$

then

(2) Not to be confused with the intersection of $G_{1}$ and $G_{2}$ which will be denoted by $G_{1} \cap G_{2}$. The union will be denoted by $G_{1} \cup G_{2}$, the complement of $G$ by $G^{\prime}$, and the boundary of $G$ by $\partial G$.

( $\left.{ }^{3}\right)$ Schmidli obtained this for $\psi(r)=1 / r^{1+\epsilon}$ by showing that $\phi$ is then continuous on $\partial D(f)$. The more general case requires no alteration in his proof. R. P. Boas has observed that if (2.5) holds; then $\phi(w)$ is uniformly bounded outside $D(f)$, and the formula (2.6) may be obtained from (2.4) by shrinking $\Gamma$. 


$$
f(z)=\frac{1}{2 \pi i} \int_{\partial D(f)} e^{z w} \phi(w) d w .
$$

We turn now to complex-valued functionals $T$ defined on $K$. Let $g$ be any entire function, and define $T$ by

$$
T(f)=\frac{1}{2 \pi i} \int_{\Gamma} g(w) \phi(w) d w
$$

where $\phi$ is the Borel transform of $f$, and $\Gamma$ encloses $D(f)$. Since the Borel transform is linear, so is $T$. In the present paper, only functionals $T$ of the above form will be discussed $\left({ }^{4}\right)$. The function $g$ is referred to as a generating function for $T$. If $f$ is chosen so that $f(z)=e^{w z}$, then $T(f)=g(w)$; thus, $T(f)$ can be formally described as $\left.g(d / d z) f(z)\right|_{z=0}[3]$.

3. Interpolation series. Let $\left\{T_{n}\right\}$ be a sequence of linear functionals, defind on all or part of $K$. A class $C \subset K$ is said to be a uniqueness class for $\left\{T_{n}\right\}$ if $T_{n}(f)=0$ for all $n$ implies $f=0$ for any $f \in C$. Thus, a function of $C$ is determined uniquely by the sequence of complex numbers $\left\{T_{n}(f)\right\}$. Suppose that it is possible to find a sequence of functions $\left\{u_{n}(z)\right\}$ which are orthogonal to $\left\{T_{n}\right\}$ in the sense that $T_{n}\left(u_{m}\right)=0$ for all $n \neq m$, while $T_{n}\left(u_{n}\right)=1$. Then any function $f$ may be represented by the formal series

$$
\sum_{0}^{\infty} u_{n} T_{n}(f)
$$

Such a series will be called an interpolation series for $\left\{T_{n}\right\}$.

We will discuss classes of functions $f$ for which the interpolation series is convergent, or is summable to $f$. Such classes must clearly be subclasses of the corresponding uniqueness class; this enables us to prove uniqueness theorems, which, however, can not be expected to be best possible, since there is reason to believe that a uniqueness class need not be an expansion class.

The argument for convergence is familiar $[1 ; 10]$. If $f(z)=e^{z w}$, then $T_{n}(f)=g_{n}(w)$, the generating function of $T_{n}$, and formally

$$
e^{z w}=\sum_{0}^{\infty} u_{n}(z) g_{n}(w) \text {. }
$$

If this converges uniformly for all $w$ on a simple contour $\Gamma$ and for all $z$, and $f$ is such that $D(f)$ lies in the interior of $\Gamma$, then using (2.4) and integrating termwise,

$$
f(z)=\sum_{0}^{\infty} u_{n}(z) \frac{1}{2 \pi i} \int_{\Gamma} g_{n}(w) \phi(w) d w=\sum_{0}^{\infty} u_{n}(z) T_{n}(f),
$$

(4) If $K$ is given the weak topology in which $f_{n} \rightarrow f$ whenever $\lim f_{n}(z)=f(z)$ for all $z$, then any continuous linear functional $T$ has the representation (2.7). See also [3]. 
convergent for all $z$.

If $f$ satisfies the stronger growth conditions required to apply the Schmidli representation, we can say more: if (3.2) holds uniformly on $\Gamma$, and if $f$ is such that $f \in K[\psi(r), h(\theta, f)]$ where $\int^{\infty} \psi(r) d r<\infty$, and $D(f)$ lies in the closed interior of $\Gamma$, then the interpolation series again converges to $f(z)$.

Turning to summability, we digress to describe the familiar notion of $E$-summability, of which Borel exponential means and Mittag-Leffler are special cases. Let $E(z)=\sum_{0}^{\infty} d_{n} z^{n}$ be an entire function, not a polynomial, with $d_{n} \geqq 0$. If $\left\{s_{n}\right\}$ is a sequence of complex numbers with $\left|s_{n}\right|^{1 / n}=O(1)$, then the series $\sum_{0}^{\infty} d_{n} s_{n} \lambda^{n}$ converges for all $\lambda, 0 \leqq \lambda<\infty$, to a function $H(\lambda)$. Consider the "mean," $\sigma(\lambda)=H(\lambda) / E(\lambda)$. If $\lim _{\lambda \rightarrow \infty} \sigma(\lambda)=S$ exists, then we write $(E)-\lim s_{n}=S$. This defines a totally regular method of summability. We use $(E)-\sum_{0}^{\infty} a_{n}$ for $(E)$-lim $s_{n}$ where $s_{n}=\sum_{0}^{\infty} a_{k}$. These methods are of particular importance when applied to power series. Let $E$ denote an open set associated with $E(z)$ such that $\lim _{\lambda \rightarrow \infty} E(z \lambda) / E(\lambda)=0$, uniformly in any compact subset of $E$. The importance of the set $E$ is that $(E)-\sum_{0}^{\infty} z^{n}=1 /(1-z)$ for all $z$ in $E$, and the summability is uniform in any compact subset of $E$. This can be extended to a general function $f$ and its Taylor series. The following proof differs slightly from that usually given for Borel summability [7].

THEOREM 3.1. Let $f(z)$ be regular in a region $R$ containing the origin. Then,

$$
f(z)=(E)-\sum_{0}^{\infty} f^{(n)}(0) z^{n} / n !
$$

uniformly in any closed compact subset of $\left(R^{\prime} \cdot E^{\prime}\right)^{\prime}$, the E-star of $R$.

If $z \in E$ and $0 \leqq \mu \leqq 1$, then $|E(\mu z \lambda) / E(\lambda)| \leqq|E(\mu z \lambda) / E(\lambda \mu)|=o(1)$ as $\lambda$ increases, so that $E$ may be assumed to be a star set. It follows that $G=\left(R^{\prime} \cdot E^{\prime}\right)^{\prime}$ is also a star set. Let $G_{0}$ be any compact subset of $G$. We can then choose an open set $E_{0}$ whose closure is a compact subset of $E$, and a region $R_{0} \subset R$ such that $G_{0} \check{C}\left(R_{0}^{\prime} \cdot E_{0}^{\prime}\right)^{\prime}$. In fact, since $G$ is a star set, we can take $R_{0}$ to be simply connected, and its boundary $\Gamma=\partial R_{0}$ as a simple contour. Since 1 does not belong to $E$, and therefore not to $E_{0},\left(R_{0}^{\prime} \cdot E_{0}^{\prime}\right)^{\prime} \subset R_{0}$ and $G_{0} \subset R_{0}$. For any $z$ in $G_{0}$, we have

$$
\begin{gathered}
s_{n}(z)=\sum_{0}^{n} f^{(k)}(0) z^{k} / k !=f(z)-\frac{1}{2 \pi i} \int_{\Gamma} \frac{f(t)}{t-z}\left(\frac{z}{t}\right)^{n+1} d t, \\
f(z)-\sigma(z, \lambda)=\frac{1}{2 \pi i} \int_{\Gamma} \frac{f(t)}{t-z} \frac{z}{t} \frac{E(\lambda z / t)}{E(\lambda)} d t .
\end{gathered}
$$

Since $G_{0} \subset\left(R_{0}^{\prime} \cdot E_{0}^{\prime}\right)^{\prime}$ and $\Gamma \subset R_{0}^{\prime}, z / t$ lies in $E_{0}$ for all $z$ in $G_{0}$ and $t$ on $\Gamma$. Thus

$$
\lim _{\lambda \rightarrow \infty} \frac{E(\lambda z / t)}{E(\lambda)}=0
$$


uniformly in $z$ and $t$. Moreover, $f(t)$ is bounded on $\Gamma$, and $|t-z|$ is bounded away from zero, since $G_{0}$ is a compact subset of $R_{0}$. Hence, $\lim _{\lambda \rightarrow \infty}|f(z)-\sigma(z, \lambda)|=0$ uniformly in $G_{0}$.

If we choose $E(z)$ as $e^{z}$, then $E$ is the set of all $z, \Re(z)<1$, and $\left(R^{\prime} \cdot E^{\prime}\right)^{\prime}$ is easily seen to be the Borel polygon of $R$. If $E(z)$ is taken as $\sum(z / \log (n+2))^{n}$, then $E$ is the whole plane with the half line $y=0, x \geqq 1$ deleted, and the $E$-star of $R$ becomes simply the (Mittag-Leffler) star of $R$. Since the interval $[1,+\infty]$ always lies outside an $E$ set, the $E$-star of $R$ is a star set lying in the star of $R$.

Returning to the discussion of interpolation series, we can replace convergence by summability. If, for all $z$,

$$
e^{z w}=(E)-\sum_{0}^{\infty} u_{n}(z) g_{n}(w)
$$

uniformly on $\Gamma$, then for all $f$ such that $D(f)$ lies in the interior of $\Gamma$, and for all $z$,

$$
f(z)=(E)-\sum_{0}^{\infty} u_{n}(z) T_{n}(f)
$$

If $f$ is such that $f \in K[\psi(r), h(\theta, f)]$ where $\int{ }^{\infty} \psi(r) d r<\infty$, and $D(f)$ lies in the closed interior of $\Gamma$, then (3.4) still holds.

We now assume that the generating functions of $\left\{T_{n}\right\}$ are of a special form. We suppose that $g_{n}(w)=[\zeta(w)]^{n}$ where $\zeta(w)$ is an entire function. Let $\Omega_{w}$ be a set containing the origin in which $\zeta(w)$ is univalent, and let $\Omega_{\zeta}$ be the image of $\Omega_{w}$ under $\zeta=\zeta(w)$; let $\Delta_{\zeta}$ be the largest open circle $|\zeta|<R$ in $\Omega_{\zeta}$, and let $\Delta_{w}$ be its image under $w=w(\zeta)$ in $\Omega_{w}$; let $\Omega_{\zeta}^{*}$ be the $E$-star of $\Omega_{\xi}$, and let $\Omega_{w}^{*}$ be its image in $\Omega_{w}$. The central theorem of [4], proved there for a general kernel $\gamma(z)$ and stated below for the special kernel $\gamma(z)=e^{z}$ becomes:

THEOREM 3.2. The formal interpolation series $\sum_{0}^{\infty} u_{n}(z) T_{n}(f)$ converges to $f(z)$ for all $f$ such that $D(f) \subset \Delta_{w}$, and is $E$ summable to $f(z)$ for all $f$ with $D(f) \subset \Omega_{w}^{*}$.

To obtain the sharper convergence forms, we must have $\sum_{0}^{\infty} u_{n}(z)[\zeta(w)]^{n}$ converging uniformly to $e^{z w}$ on $\Gamma$. This usually requires a separate treatment; however, since $e^{z w}$ is a regular function of $\zeta$ for $\zeta$ in $\Omega_{\zeta}$, we can state that if $\lim u_{n}(z) R^{n}=0, \sum u_{n}(z) \zeta^{n}$ converges uniformly on all arcs of $|\zeta|=R$ where it is regular, considered as a function of $z$. In the succeeding sections, we take up special interpolation series and apply the results of this section in detail.

4. Newton series. To obtain this, we define the functionals $\left\{T_{n}\right\}$ by $T_{n}(f)=\Delta^{n} f(0)=(-1)^{n} \sum_{0}^{n} C_{n, k}(-1)^{k} f(k)$. The generating functions $g_{n}(w)$ have the form $[\zeta(w)]^{n}$ where $\zeta(w)=e^{w}-1$, and the functions $u_{n}$ are the polynomials $u_{n}(z)=C_{z, n}=z(z-1)(z-2) \cdots(z-n+1) / n !$. For $\Omega_{w}$, we take the 
strip $|v|<\pi ; \Omega_{\zeta}$ is then the $\zeta$-plane with $[-\infty,-1]$ deleted. The circle $\Delta_{\zeta}$ is $|\zeta|<1$, and $\Delta_{w}$ is the set lying in $\Omega_{w}$ and satisfying $\left|e^{x}-1\right|<1$; the boundary of $\Delta_{w}$ has the equation $u=\log (2 \cos v)$. Applying Theorem 3.2 for convergence, we at once have:

THEOREM 4.1. If $D(f) \subset \Delta_{w}$, the subset of $\Omega_{w}$ containing zero and bounded by $u=\log (2 \cos v)$, then the Newton series

$$
\sum_{0}^{\infty} C_{z, n} \Delta^{n} f(0)
$$

converges to $f(z)$ for all $z$.

The condition $D(f) \subset \Delta_{w}$ may also be expressed in the form: $k(\theta, D(f))$ $<k\left(\theta, \Delta_{w}\right)$ for all $\theta$. Since $k(\theta, D(f))=h(\theta, f)$ and since [18, vol. 1, part III, no. 114]

$$
k\left(\theta ; \Delta_{w}\right)=\cos \theta \log (2 \cos \theta)+\theta \sin \theta
$$

for all $\theta,|\theta|<\pi / 2$, we can rephrase this theorem [6].

Corollary 1. If $h(\theta, f)<\cos \theta \log (2 \cos \theta)+\theta \cos \theta$ for all $|\theta|<\pi / 2$, then the Newton series (4.1) converges to $f(z)$ for all $z$.

Since the least value of $k\left(\theta, \Delta_{w}\right)$ is $\log 2$, the condition $f \in K[A], A<\log 2$, is sufficient to insure the convergence of the Newton series $[24$, p. 55].

We can also obtain sharper results on convergence. Since $\lim _{n \rightarrow \infty}\left|C_{z, n}\right|=0$ for all $z$ such that $\Re(z)>1$, the series $\sum C_{z, n} \zeta^{n}$ converges uniformly in any closed subset of the closed circle $|\zeta| \leqq 1$ not containing the point -1 . If $f \in K$, the set $D(f)$ is bounded; thus, if $D(f)$ lies in the closure of $\Delta_{w}$, its image under $\zeta=e^{w}-1$ is a compact subset of the closed unit circle and does not contain -1 . Hence, if $D(f)$ lies in the closure of $\Delta_{w}$ and $f$ obeys condition (2.5), we can again infer the convergence of the Newton series; the resulting theorem is not as strong as has been obtained by Nörlund who assumed only that $f$ was regular in a half plane [15].

ThEOREM 4.2. If $f \in K[\psi(r), \theta \sin \theta+\cos \theta \log (2 \cos \theta)]$ where $\int{ }^{\infty} \psi(r) d r<\infty$, then the Newton series (4.1) converges to $f(z)$ for all $z, \Re(z)>-1$.

We now consider summability of the Newton series. In applying Theorem 3.2 , we first observe that the $E$-star of $\Omega_{\zeta}$ is simply $\{-1\} \cdot E$, which we write as $-E$; applying the general theorem, we have:

Theorem 4.3. If $D(f)$ lies in the image of the set $-E$ under $w=\log (1+\zeta)$, then

$$
f(z)=(E)-\sum_{0}^{\infty} C_{z, n} \Delta^{n} f(0)
$$


If we specialize this to Borel summability by choosing $E(z)=e^{z}$, then $-E$ is the half plane $\Re(z)>-1$, and its image is the strip $|v|<\pi / 2\left(^{5}\right)$.

Corollary 1. If $f \in K(a, c)$ with $c<\pi / 2$, then

$$
f(z)=(B)-\sum_{0}^{\infty} C_{z, n} \Delta^{n} f(0) .
$$
$\Omega_{w}$.

For Mittag-Leffler summability, $-E$ is exactly $\Omega_{\zeta}$, and $\Omega_{w}{ }^{*}$ is therefore

Corollary 2. If $f \in K(a, c)$ with $c<\pi$, then

$$
f(z)=(M L)-\sum_{0}^{\infty} C_{z, n} \Delta^{n} f(0) .
$$

Convergence may also be established by the addition of Tauberian conditions. Two examples of this will be sufficient illustration of the method.

Corollary 3. If $f \in K(a, c), c<\pi / 2$, and $\Delta^{n} f(0)=o\left(1 / n^{1 / 2}\right)$ then the Newton series (4.1) converges to $f(z)$ for all $z, \Re(z)>-1$.

Corollary 4. If $f \in K(a, c), c<\pi$, and $(-1)^{n} \Delta^{n} f(0) \geqq 0$ for $n=0,1, \cdots$. then the Newton series (4.1) converges to $f(z)$ for all $z$.

The first of these follows from Corollary 1; the Newton series is Borel summable for $z=-1$, and $C_{-1, n}=(-1)^{n}$ so that the terms of this series satisfy the Tauberian condition $o\left(1 / n^{1 / 2}\right)$. Applying a theorem of Hardy [14], the series converges; it then follows that since the Newton series converges for $z=-1$, it also converges for all $z$ with $\Re(z)>-1$.

The second arises in a similar manner from Corollary 2 ; the series is $M L$ summable for $z=-k$, and $C_{-k, n} \Delta^{n} f(0)$ is by hypothesis positive for each $n$. Since $M L$ summability is totally regular, the series must in fact converge for $z=-k$, and since this holds for any $k$, we have convergence for all $z$.

5. Stirling series. To obtain one form of this interpolation series, we take $T_{n}(f)=\Delta^{n} f(-n / 2)=(-1)^{n} \sum_{0}^{n} C_{n, k}(-1)^{k} f(k-n / 2)$. The generating function $g_{n}(w)$ is $\left(e^{w}-1\right)^{n} e^{-n w / 2}$ which is $(\zeta(w))^{n}$ for $\zeta(w)=2 \sinh (w / 2)$. The functions $u_{n}(z)$ are

$$
u_{0}(z)=1, \quad u_{n}(z)=\frac{z}{n} C_{z-1+n / 2, n-1}
$$

(5) Using results of Dienes [8], it may be shown that $e^{z w}=(B)-\sum C_{z, n}[\zeta(w)]^{n}$, uniformly in compact subsets of $\Re(\zeta)=1$, if $\Re(z)>0$. We can then obtain a stronger form of Corollary 1. If $|f(z)| \leqq \psi(r)$ exp $\{a|x|+(\pi / 2)|y|\}$ where $\int \psi(r) d r<\infty$, then $f(z)=(B)$ $-\sum C_{z, n} \Delta^{n} f(0)$ for all $z$ with $\Re(z)>0$. This method will not work for $M L$ summability, since we cannot expect the expansion of $e^{z w}$ to be summable on the boundary of $\Omega_{\zeta}$; for this reason no attempt will be made in the present paper to obtain sharper forms of the summability theorems. 
$\zeta(w)$ is univalent in the strip $|v|<\pi$, which we may therefore take to be the set $\Omega_{w} ; \Omega_{\zeta}$ becomes the $\zeta$-plane with cuts from $2 i$ to $+\infty i$, and from $-2 i$ to $-\infty i$ deleted. The circle $\Delta_{\zeta}$ is $|\zeta|<2$, and the set $\Delta_{w}$ is the subset of $\Omega_{w}$ described by $|\sinh (w / 2)|<1$. The supporting function of $\Omega_{w}$ is [18, III, no 115]:

$$
k\left(\theta, \Delta_{w}\right)=\left\{\begin{array}{cc}
2 \cos \theta \log \left[2^{1 / 2} \cos \theta+(\cos 2 \theta)^{1 / 2}\right] \\
+2 \sin \theta \sin ^{-1}\left(2^{1 / 2} \sin \theta\right), & |\theta| \leqq \pi / 4, \\
\pi|\sin \theta|, & \pi / 4 \leqq|\theta| \leqq \pi / 2, \\
k\left(\theta \pm \pi, \Delta_{w}\right), & \pi / 2 \leqq|\theta| \leqq \pi
\end{array}\right.
$$

Applying Theorem 3.2, we obtain the following result first proved by Nörlund using different methods [15].

Theorem 5.1. If $h(\theta, f)<k\left(\theta, \Delta_{w}\right)$, given by (5.1), then the Stirling series

$$
f(0)+\sum_{1}^{\infty} \frac{z}{n} C_{z-1+n / 2, n-1} \Delta^{n} f(-n / 2)
$$

converges to $f(z)$ for all $z$.

Since $\left|u_{n}(z)\right| \leqq O\left(2^{-n} n^{-3 / 2}\right)$, the expansion of $e^{z w}$ in powers of $\zeta$ converges for all $\zeta,|\zeta| \leqq 2$. This gives at once the following extension of a result of Schmidli [20].

Theorem 5.2. If $f \in K\left[\psi(r), k\left(\theta, \Delta_{w}\right)\right]$ where $\int^{\infty} \psi(r) d r<\infty$, then the Stirling series (5.2) converges to $f(z)$ for all $z$.

The least value of $k\left(\theta, \Delta_{w}\right)$ is $\log \left(3+2^{3 / 2}\right)$; thus, the condition that $f \in K[A]$ with $A<\log \left(3+2^{3 / 2}\right)$ or $f \in K\left[\psi(r), \log \left(3+2^{3 / 2}\right)\right]$ is sufficient to insure convergence. (The first of these is due to Pólya [17].)

For Borel summability we consider the corresponding $E$-star of $\Omega_{\xi}$ which in this case is the strip $|\Im(\zeta)|<2$, the Borel polygon of $\Omega_{\zeta}$. Its image $\Omega_{w}{ }^{*}$ is the set bounded by the curves $\cosh (u / 2) \sin (v / 2)=1$. We therefore have Borel summability whenever $D(f)$ lies in the interior of this set. We cannot make use of the supporting function of this set as a bound on $h(\theta, f)$ since this set is not convex. However, since $\cos t \cosh t \leqq 1$ for all $t, 0 \leqq t \leqq \pi / 2$, the square $S$ with vertices $\pi,-\pi, \pi i,-\pi i$ lies in $\Omega_{w}^{*}$, and is convex. Its supporting function is seen to be

$$
k(\theta, S)= \begin{cases}\pi|\cos \theta| & \text { for }|\theta|<\pi / 4 \text { or }|\theta-\pi|<\pi / 4, \\ \pi|\sin \theta| & \text { for } \pi / 4 \leqq|\theta| \leqq 3 \pi / 4\end{cases}
$$

THEOREM 5.3. If $D(f)$ lies in the region bounded by the curves cosh $(u / 2)$ $\cdot \sin (v / 2)=1$, or if $h(\theta, f)<k(\theta, S)$, then

$$
f(z)=(B)-\left\{f(0)+z / n C_{z-1+n / 2, n-1} \Delta^{n} f(-n / 2)\right\} .
$$


Corollary. If $f \in K\left[\pi / 2^{1 / 2}\right]$, then the Stirling series (5.2) is Borel summable to $f(z)$ for all $z$.

For the circle $|w|=\pi / 2^{1 / 2}$ lies in $S$.

Theorem 5.4. If $f \in K(a, c), c<\pi$, then the Stirling series (5.2) is $M L$ summable to $f(z)$ for all $z$.

6. Generalized difference series. If we take $T_{n}(f)=\Delta^{n} f(\beta n)=(-1)^{n}$ - $\sum_{0}^{n} C_{n, k}(-1)^{k} f(\beta n+k)$, we obtain a general class of interpolation series of which the Newton and Stirling discussed in the prceeding sections are special cases. The generating functions are $g_{n}(w)=\left(e^{w}-1\right)^{n} e^{\beta n w}$, and $\zeta(w)=e^{\beta w}\left(e^{w}-1\right)$. The functions $u_{n}(z)$ are defined by

$$
u_{0}(z)=1, \quad u_{n}(z)=(z / n) C_{z-\beta n-1, n-1} .
$$

We shall study these series for $\beta>0$. The special case arising from $\beta=1$ has been used by Selberg, and studied briefly by Gelfond $[10 ; 22]$.

LEMMA. $\zeta(w)=e^{\beta w}\left(e^{w}-1\right)$ is univalent in the region $\Omega_{w}$ containing the origin and bounded by the curve $\Gamma$ whose equation is $u=\log \sin \beta v-\log \sin (\beta+1) v$.

Computation shows that $\cos (\arg \zeta)=-1$ on $\Gamma$; angles at $w=\log (\beta /(1+\beta))$ are doubled.

This gives us the set $\Omega_{w}$. Its boundary $\Gamma$ is convex, opens to the right, cuts the $v$ axis at $w= \pm \pi i /(2 \beta+1)$, the $u$ axis at $w=\log (\beta /(1+\beta))$, and has the lines $v= \pm \pi /(\beta+1)$ for asymptotes. The image of $\Omega_{w}$ under $\zeta=\zeta(w)$ is the $\zeta$-plane, with a cut from $\zeta_{0}=\zeta(\log (\beta /(1+\beta)))$ to $-\infty$ deleted, which is therefore the set $\Omega_{\zeta}$.

Applying Theorem 3.2 for $M L$ summability, we have:

THEOREM 6.1. If $D(f)$ lies in the region $\Omega_{w}$ bounded by the curve $\Gamma$ whose equation is $u=\log \sin \beta v-\log \sin (\beta+1) v$, then

$$
f(z)=(M L) \cdot\left\{f(0)+\sum_{1}^{\infty} \frac{z}{n} C_{z-\beta n-1, n-1} \Delta^{n} f(\beta n)\right\} .
$$

It can be shown that the circle $|w|<\log (1+\beta) / \beta$ lies inside $\Omega_{w}$ if $\beta$ is greater than $\beta_{0}(\sim 1 / 12)$, the positive root of $\log (1+\beta) / \beta=3 /(2 \beta+1)$. This is the value for which the circle described becomes the circle of curvature. We then conclude that the expansion (6.2) holds for all $f \in K[A]$ with $A<\log (1+\beta) / \beta$.

For special choices of $\beta$, we can obtain more detailed results. For example, in the case studied by Selberg and Gelfond, $\beta=1, \Gamma$ has the equation: $u=-\log (2 \cos v)$. then

Corollary. If $h(\theta, f)<(\pi-\theta) \sin \theta+\cos \theta \log (-2 \cos \theta)$ for $\pi / 2 \leqq|\theta| \leqq \pi$, 


$$
f(z)=(M L)-\left\{f(0)+\sum_{1}^{\infty} \frac{z}{n} C_{z-n-1, n-1} \Delta^{n} f(n)\right\} .
$$

We can also discuss convergence. For the special case that we are treating, $\zeta_{0}=\zeta(\log 1 / 2)=-1 / 4$, and $\Delta_{\zeta}$ is $|\zeta|<1 / 4$ and $\Delta_{w}$ is the subset of $\Omega_{w}$ satisfying $\left|e^{w}\left(e^{w}-1\right)\right|<1 / 4$.

THEOREM 6.2. If $D(f)$ lies in the region contained in $\Omega_{w}$ and bounded by $\left|e^{w}\left(e^{w}-1\right)\right|=1 / 4$ - for example, if $f \in K[A]$ with $A<\log \left(1+2^{1 / 2}\right) / 2$-then

$$
f(0)+\sum_{1}^{\infty} \frac{z}{n} C_{z \rightarrow n-1, n-1} \Delta^{n} f(n)
$$

converges to $f(z)$ for all $z$.

In general, $u_{n}(z)$, given by (6.1), satisfies

$$
\left|u_{n}(z)\right|=O(1) n^{-3 / 2}\left[\beta^{-\beta}(\beta+1)^{1+\beta}\right]^{n}
$$

so that $\sum u_{n}(z) \zeta^{n}$ converges uniformly for $|\zeta|=\left|\zeta_{0}\right|$.

Theorem 6.3. If $D(f)$ lies in the closure of $\Delta_{w}$, and if $f \in K[\psi(r), h(\theta, f)]$ where $\int \psi(r) d r<\infty$, then (6.3) converges to $f(z)$.

7. Abel series. For this, we take $T_{n}(f)=f^{(n)}(n)$, and $g_{n}(w)=w^{n} e^{n w}$ $=[\zeta(w)]^{n}$ with $\zeta(w)=w e^{w}$. The functions $u_{n}$ are given by

$$
u_{0}(z)=1, \quad u_{n}(z)=z(z-n)^{n-1} / n ! .
$$

LEMмA. $\zeta(w)=w e^{w}$ is univalent in the region containing the origin and bounded by the curve whose equation is

$$
\rho=\frac{\pi-|\phi|}{\sin |\phi|}
$$

where $w=\rho e^{i \phi}$.

Computation shows that arg $\left(w e^{w}\right)=\pi$ when $\phi>0$ and $\rho=(\pi-\phi) / \sin \phi$, and $-\pi$ when $\phi<0$ and $\rho=-(\pi+\phi) / \sin \phi$. Angles at $w=-1, \zeta=-e^{-1}$ are doubled.

This gives us the set $\Omega_{w}$; its boundary, $\Gamma$, opens to the right, cuts the real axis at -1 , and has the lines $v= \pm \pi$ for asymptotes. Under $\zeta=w e^{w}, \Omega_{w}$ is mapped onto the $\zeta$-plane with the interval $\left[-e^{-1},-\infty\right]$ deleted; this set is then $\Omega_{\zeta}$. The circle $\Delta_{\zeta}$ is $|\zeta|<1 / e$, and $\Delta_{w}$ is the subset of $\Omega_{w}$ defined by $\left[w e^{1+w} \mid<1\right.$, a convex set. Computing the supporting function of $\Delta_{w}$, we have

$$
k\left(\theta, \Delta_{w}\right)= \begin{cases}\sin 2 \psi / 2 \sin \theta & \text { for } 0 \leqq|\theta| \leqq 3 \pi / 4, \\ |\cos \theta| & \text { for } 3 \pi / 4<|\theta| \leqq \pi,\end{cases}
$$

where 


$$
0=\log \{\cos \psi / \sin \theta\}+\sin \psi(\sin \psi+\cot \theta \cos \psi) .
$$

Applying Theorem 3.2, we obtain the following theorem on convergence.

THEOREM 7.1. If $D(f) \subset \Delta_{w}$, or if $h(\theta, f)<k\left(\theta, \Delta_{w}\right)$, given by (7.2), then the Abel series

$$
f(0)+\sum_{1}^{\infty} \frac{z(z-n)^{n-1}}{n !} f^{(n)}(n)
$$

converges to $f(z)$ for all $z$.

This result is not new $[9 ; 11]$. The least value of $k\left(\theta, \Delta_{w}\right)$ is assumed for $\theta=0$, and is the positive root of $x e^{1+x}=1$ which is approximately .278 . Thus, we have convergence of the Abel series if $f \in K[A]$ where $A<.278[9 ; 12]$.

Stronger results follow as before; since $\left|u_{n}(z)\right| \leqq O(1) e^{n} n^{-3 / 2}$ the series $\sum u_{n}(z) \zeta^{n}$ converges uniformly to $e^{z w}$ for all $\zeta$ with $[\zeta] \leqq e^{-1}$. This leads to the following slight extension of a result due to Schmidli [20].

THEOREM 7.2. If $f \in K\left[\psi(r), k\left(\theta, \Delta_{w}\right)\right]$ where $\int^{\infty} \psi(r) d r<\infty$, then the Abel series (7.3) converges to $f(z)$ for all $z$.

For Mittag-Leffler summability, $\Omega_{\xi}^{*}$ is $\Omega_{\zeta}$ since this is already a star set, and $\Omega_{w}{ }^{*}$ is the convex set $\Omega_{w}$, bounded by the curve (7.1). Its supporting function is

$$
k\left(\theta, \Omega_{w}\right)=\beta(\sin \theta-\cos \theta \cot \beta),
$$$$
\pi / 2 \leqq|\theta| \leqq \pi
$$

where

$$
\tan \theta=\cot \beta-\beta \csc ^{2} \beta .
$$

Theorem 7.3. If $h(\theta, f)<k\left(\theta, \Omega_{w}\right)$, then the Abel series (7.3) is ML summable to $f(z)$ for all $z$.

The minimum value of $k\left(\theta, \Omega_{w}\right)$ is 1 , achieved for $\theta=\pi$. Hence, the condition $f \in K[A]$, with $A<1$, is sufficient for $M L$ summability.

Borel summability, or more generally $E$ summability, can also be discussed as before. The $E$-star of $\Omega_{\zeta}$ is simply $\left\{-e^{-1}\right\} \cdot E$ and $\Omega_{w}^{*}$ is the image of this set under $w=w(\zeta)$. Hence, if $D(f)$ lies in this set, the Abel series (7.3) is $E$ summable to $f(z)$. However, the region $\Omega_{w}{ }^{*}$ will in general be quite complicated in description. Thus, for Borel, its boundary is the curve whose equation is $\log \rho+\rho \cos \phi+\log \cos (\rho \sin \phi+\phi)=-1$.

8. Generalized Newton series. Formally, the following expansion is valid:

$$
e^{z v}=\beta^{z} \sum_{0}^{\infty} C_{z, n} \beta^{-n}\left(e^{w}-\beta\right)^{n} .
$$


This converges for all $z$ when $\left|e^{w}-\beta\right|<|\beta|$, and converges for all $z$ such that $\Re(z)>-1$ when $\left|e^{w}-\beta\right| \leqq|\beta|$ and $w$ is finite. This expansion yields an interpolation series which reduces to the Newton series when $\beta=1$. We take $\beta$ real and positive. If $g_{n}(w)=\left(e^{w}-\beta\right)^{n}$ are chosen as generating functions for the functionals $\left\{T_{n}\right\}$, then

$$
T_{n}(f)=(-\beta)^{n} \sum_{0}^{n} C_{n, k}(-1 / \beta)^{k} f(k) .
$$

Since $\zeta(w)=e^{w}-\beta, \Omega_{w}$ is again the strip $|v|<\pi$ and $\Omega_{\zeta}$ is the $\zeta$-plane, with the interval $[-\beta,-\infty]$ deleted; the functions $u_{n}(z)$, from (8.1), are seen to be $\beta^{z} C_{z, n} \beta^{-n}$. The results for Borel and Mittag-Leffler summability are the same for this series as for the Newton series, since the corresponding sets $\Omega_{w}{ }^{*}$ are the same.

\section{THEOREM 8.1. The series}

$$
\beta^{z} \sum_{0}^{\infty} C_{z, n} \beta^{-n} T_{n}(f)
$$

is Borel summable to $f(z)$ for all $z$ if $f \in K(a, c)$ with $c<\pi / 2$, and is MittagLeffler summable to $f(z)$ if $f \in K(a, c), c<\pi$.

The interpolation series is convergent for a wider class of functions than the Newton series, if $\beta>1$. The circle $\Delta_{\zeta}$ is $|\zeta|<\beta$, and $\Delta_{w}$ is the set containing the origin and bounded by the curve $u=\log (2 \beta \cos v)$. The supporting function of $\Delta_{w}$ is given by

$$
k\left(\theta, \Delta_{w}\right)=\theta \sin \theta+\cos \theta \log (2 \beta \cos \theta) .
$$

Theorem 8.2. If $h(\theta, f)<k\left(\theta, \Delta_{w}\right)$, then the generalized Newton series (8.2) converges to $f(z)$ for all $z$.

As $\beta$ increases, the set $\Delta_{w}$ approaches the strip $|v|<\pi / 2$. If $f \in K(a, c)$ with $c<\pi / 2$, then $D(f)$ is a compact subset of this open strip, and thus a subset of $\Delta_{w}$ for sufficiently large $\beta$.

COROLlaRy. If $f \in K(a, c)$, with $c<\pi / 2$, then for sufficiently large $\beta$, the series (8.2) converges to $f(z)$ for all $z$.

Making use of the convergence of (8.1) on the boundary of $\Delta_{\zeta}$, as observed above, we can also obtain the corresponding stronger convergence form.

THEOREM 8.3. If $f \in K[\psi(r), \theta \sin \theta+\cos \theta \log (2 \beta \cos \theta)]$ where $\int \psi(r) d r<\infty$, then the series (8.2) converges to $f(z)$ for all $z$ with $\Re(z)>-1$.

9. Lidstone series. This well known series arises from the choice of $g_{2 n}(w)=w^{2 n}, g_{2 n+1}(w)=e^{w} w^{2 n}$, which gives $T_{2 n}(f)=f^{(2 n)}(0), T_{2 n+1}(f)=f^{(2 n)}(1)$. The generating functions are not of the form $[\zeta(w)]^{n}$ so that Theorem 3.2 does 
not apply; however, the general method of $\S 3$ may still be used. We seek an expansion of the form

$$
e^{z w}=\sum_{0}^{\infty} u_{n}(z) g_{n}(w)
$$

Requiring that the $g_{n}$ have the prescribed form, we write

$$
\begin{aligned}
e^{z w} & =F_{1}\left(w^{2}\right)+e^{w} F_{2}\left(w^{2}\right) \\
& =\frac{\sinh (1-z) w}{\sinh w}+e^{w} \frac{\sinh z w}{\sinh w}
\end{aligned}
$$

so that

$$
u_{2 n}(z)=\Lambda_{n}(1-z), \quad u_{2 n+1}(z)=\Lambda_{n}(z)
$$

where $\Lambda_{n}$ is a polynomial of degree $2 n+1$, determined by the formal identity [25]:

$$
\frac{\sinh z w}{\sinh w}=\sum_{0}^{\infty} \Lambda_{n}(z) w^{2 n}
$$

Accordingly, the formal Lidstone two-point expansion is

$$
f(z)=\sum_{0}^{\infty} \Lambda_{n}(1-z) f^{(2 n)}(0)+\sum_{0}^{\infty} \Lambda_{n}(z) f^{(2 n)}(1) .
$$

In (9.2), the only singularities of $F_{1}$ and $F_{2}$ occur at the points $\pm \pi n i$, $n \geqq 1$; the series (9.1) will then converge for $|w|<\pi$, be Borel summable for $|v|<\pi$, and be $M L$ summable for $u \neq 0$, or $|v|<\pi$.

Theorem 9.1. If $f \in K[A], A<\pi$, the Lidstone series (9.3) converge to $f(z)$ for all $z$. If $f \in K(a, c), c<\pi$, then they are Borel summable.to $f(z)$ for all $z$. If $D(f)$ does not contain any points of the lines $u=0,|v| \geqq \pi$, then the series are Mittag-Leffler summable to $f(z)$ for all $z$.

The convergence result is not as strong as can be proved; it is known that if $f \in K\left[\eta(r) / r^{1 / 2}, \pi\right]$ where $\eta(r)=o(1)$ as $r$ increases, then the Lidstone expansion of $f$ converges [2]. This illustrates again the limitations of the present method, for it can be easily seen that the expansion of $e^{z w}$ in (9.1) does not converge on $|w|=\pi$.

The same approach can be made to the generalized $n$-point expansion and other essentially periodic type functionals $[19 ; 20]$. If $a_{1}, \cdots, a_{r}$ are distinct complex numbers, we define $\left\{T_{n}\right\}$ by $T_{r n+j}(f)=f^{(r n)}\left(a_{j}\right)$ for $j=1,2, \cdots, r$, and $n=0,1,2, \cdots$. The generating functions are given by

$$
g_{r n+j}(w)=w^{r n} \exp \left(a_{j} w\right) .
$$


We first seek an expansion of the form

$$
\begin{aligned}
e^{z w}=\sum_{0}^{\infty} u_{n}(z) g_{n}(w) & =\sum_{j=1}^{r} \sum_{n=0}^{\infty} u_{r n+j}(z) w^{r n} \exp \left(a_{j} w\right) \\
& =\sum_{j=1}^{r} F_{j}\left(w^{r}\right) \exp \left(a_{j} w\right) .
\end{aligned}
$$

Let $\sigma$ be a primitive $r$ th root of unity; then

$$
\exp \sigma^{k} z w=\sum_{j=1}^{r} F_{j}\left(w^{r}\right) \exp \left(\sigma^{k} a_{j} w\right)
$$

for $k=1,2, \cdots, r$. Solving these for the functions $F_{j}$, they are obtained as quotients of exponential polynomials. If

$$
B(w)=B\left(w ; a_{1}, \cdots, a_{r}\right)=\left|\begin{array}{ccc}
\exp \left(\sigma a_{1} w\right) & \cdots & \exp \left(\sigma a_{r} w\right) \\
\vdots & \vdots \\
\exp \left(\sigma^{r} a_{1} w\right) & \cdots & \exp \left(\sigma^{r} a_{r} w\right)
\end{array}\right|
$$

then $F_{j}(w r)=B\left(w ; a_{1}, \cdots, a_{j-1}, z, a_{j+1}, \cdots, a_{r}\right) / B(w)$, which serves to define the polynomials $u_{n}(z)$.

It is readily seen that $F_{j}$ is regular at $w=0$, and its only singularities occur at the other zeros of $B(w)$. If $\Omega_{w}^{*}$ is the star with vertices at these zeros, then the expansion

$$
f(z)=\sum_{0}^{\infty} u_{n}(z) T_{n}(f)=\sum_{j=1}^{r} \sum_{n=0}^{\infty} u_{r n+j}(z) f^{(r n)}\left(a_{j}\right)
$$

is Mittag-Leffler summable for all $f$ such that $D(f) \subset \Omega_{w}^{*}$.

10. Uniqueness theorems. In this section, we shall use the preceding results to obtain uniqueness classes for certain functionals $\left\{T_{n}\right\}$. Clearly, if there is a formal interpolation series $f(z)=\sum u_{n}(z) T_{n}(f)$, then a convergence class is also a uniqueness class. However, in most cases, the functionals $\left\{T_{n}\right\}$ are total over a much larger class of functions than that for which the interpolation series converges. Gelfond, in an effort to treat these two problems in a unified manner, was able in certain cases to determine a uniqueness class for $\left\{T_{n}\right\}$ by constructing a new sequence of functionals $\left\{T_{n}^{*}\right\}$, total over the same class and therefore having the same uniqueness class, but having a larger expansion class for the corresponding interpolation series [10].

If we observe that in order to conclude that $f=0$ if it is known that $T_{n}(f)=0$ for $n=0,1, \cdots$, it is sufficient to have the corresponding interpolation series merely summable by some regular method; then we obtain at once all of Gelfond's results by applying Theorem 3.2, using Mittag-Leffler summability. This gives the following general theorem.

THEOREM 10.1. If $g_{n}(w)=[\zeta(w)]^{n}$ are the generating functions of $\left\{T_{n}\right\}$, if 
$\Omega_{w}$ is a region of univalence of $\zeta(w)$, if $\Omega_{\zeta}$ is the image of $\Omega_{w}$ under $\zeta=\zeta(w)$, if $\Omega_{\zeta}^{*}$ is the star of $\Omega_{\zeta}$, and if $\Omega_{w}^{*}$ is its image in $\Omega_{w}$ under $w=w(\zeta)$, then the class of all $f$ such that $D(f) \subset \Omega_{w}^{*}$ is a uniqueness class for $\left\{T_{n}\right\}$.

Specialization of $\zeta(w)$ at once yields the results of Gelfond, as well as new results and extensions. We give only a few of these. $f=0$.

Corollary 1. If $f \in K(a, c), c<\pi$, and $\Delta^{n} f(0)=0$ for $n=0,1, \cdots$, then

The function $f(z)=\sin \pi z$ shows that the critical value of $c$ cannot be increased.

Corollary 2. If $f \in K(a, c), c<\pi$, and $\Delta^{n} f(-n / 2)=0$ for $n=0,1, \cdots$, then $f=0$.

The example $f(z)=z \sin \pi z$ shows that $\pi$ is again best.

COROLlaRy 3. If $D(f)$ lies in the region containing zero, and bounded by the curve $u=\log \sin \beta v-\log \sin (1+\beta) v$ for $\beta>0$, and if $\Delta^{n} f(\beta n)=0$ for $n=0,1, \cdots$, then $f=0$.

Gelfond studied the special case $\beta=1$; his result is included in the following corollary.

Corollary 4. If $h(\theta, f)<(\pi-\theta) \sin \theta+\cos \theta \log (-2 \cos \theta)$ for $\pi / 2 \leqq|\theta|$ $\leqq \pi$, and $\Delta^{n} f(n)=0$ for $n=0,1, \cdots$, then $f=0$.

Corollary 5. If $h(\theta, f)<k\left(\theta, \Omega_{w}\right)$ given by (7.4), and if $f^{(n)}(n)=0$ for $n=0,1, \cdots$, then $f=0$.

This was also obtained by Gelfond, although the function $k\left(\theta, \Omega_{w}\right)$ is incorrectly given. In particular, the class $K[A]$, with $A<1$, is a uniqueness class for these Abel functionals. The example $f(z)=z e^{-z}$ shows that this value of $A$ cannot be improved.

Corollary 6. If $f \in K(a, c), c<\pi$, and for $n=0,1, \cdots$ and some positive $\beta$,

$$
\sum_{k=0}^{n} C_{n, k}(-1 / \beta)^{k} f(k)=0,
$$

then $f=0$.

Corollary 7. If $f \in K$ and $D(f)$ does not contain any of the points $w=i v$, $|v| \geqq \pi$, and if $f^{(2 n)}(0)=f^{(2 n)}(1)=0$ for $n=0,1, \cdots$, then $f=0$.

Although more general than that usually stated, this result also follows from the general characterization theorem of Schoenberg for Lidstone series [21].

It is often possible to obtain a uniqueness class for one sequence of functionals from that for another. If, for example, $\left\{T_{n}^{*}\right\}$ is a second sequence so 
related to $\left\{T_{n}\right\}$ that if $T_{n}(f)=0$ for all $n$ implies that $T_{n}^{*}(f)=0$ for all $n$, then a uniqueness class for $\left\{T_{n}^{*}\right\}$ is also one for $\left\{T_{n}\right\}$. This is the case with $T_{n}(f)=f(n)$, and $T_{n}^{*}(f)=\Delta^{n} f(0)$. Thus, from Corollary 1 above, we obtain the following form of Carlson's theorem [13]. $f=0$.

Corollary 8. If $f \in K(a, c), c<\pi$, and if $f(n)=0$ for $n=0,1, \cdots$, then

\section{BIBLIOGRAPHY}

1. R. P. Boas Jr., Entire functions of exponential type, Bull. Amer. Math. Soc. vol. 48 (1942) pp. 839-849.

2. - Representation of functions by Lidstone series, Duke Math. J. vol. 10 (1943) pp. 239-245.

3. - Functions of exponential type (III), Duke Math. J. vol. 11 (1944) pp. 507-511.

4. R. C. Buck, Interpolation and uniqueness of entire functions, Proc. Nat. Acad. Sci. U.S.A. vol. 33 (1947; pp. 288-292.

5. F. Carlson, Sur une classe de séries de Taylor, Thesis, Uppsala, 1914.

6. - Sur les séries de coefficients binomiaux, Nova Acta Regiae Societatis Scientiarum Upsaliensis (4) vol. 4 (1915) pp. 1-61.

7. P. Dienes, The Taylor series, Oxford, 1931, p. $302 \mathrm{ff}$.

8. - Lę̧ons sur les singularités des fonctions, Paris, 1913, chap. III.

9. M. T. Eweida, $A$ note on the generalization of Taylor's expansion, Proc. Math. Phys. Soc. Egypt vol. 3 (1946) pp. 1-7.

10. A. Gelfond, Interpolation et unicité des fonctions entières, Rec. Math. (Mat. Sbornik) N.S. vol. 4 (1938) pp. 115-147.

11. W. Gontcharoff, Sur la convergence de la série d'Abel, Rec. Math. (Mat. Sbornik) N.S. vol. 42 (1935) pp. 473-483.

12. G. H. Halphen, Oeuvres, vol. 2, Paris, 1918, pp. 520-539.

13. G. H. Hardy, On two theorems of F. Carlson and S. Wigert, Acta Math. vol. 42 (1920) pp. 327-339.

14. G. H. Hardy and J. E. Littlewood, The relation between Borel's and Cesaro's methods of summability, Proc. London Math. Soc. (2) vol. 11 (1912-1913) pp. 1-16.

15. N. E. Nörlund, Lȩ̧ons sur les séries d'interpolation, Paris, 1926.

16. G. Pólya, Untersuchungen über Lücken und Singularitäten von Potenzreihen, Math. Zeit. vol. 29 (1929) pp. 549-640.

17. — Ganzwertige funktionen, Rend. Circ. Mat. Palermo vol. 40 (1915) pp. 1-16.

18. G. Pólya and G. Szegö, Aufgaben und Lehrsätze, Berlin, 1925.

19. H. Poritsky, On certain polynomial and other approximations to analytic functions, Trans. Amer. Math. Soc. vol. 34 (1932) pp. 274-331.

20. S. Schmidli, Über gewisse Interpolationsreihen, Thesis, Zurich, 1942.

21. I. J. Schoenberg, On certain two point expansions of analytic functions of exponential type, Bull. Amer. Math. Soc. vol. 42 (1936) pp. 284-288.

22. A. Selberg, Über ganzwertige ganze transzendente Funktionen, Archiv för Mathematik og Naturvidenskab vol. 44 (1941) pp. 45-52.

23. G. N. Watson, An expansion due to Abel, J. London Math. Soc. vol. 3 (1928) pp. 188192.

24. J. M. Whittaker, Interpolatory function theory, Cambridge, 1935.

25. - On Lidstone's series and two point expansions of analytic functions, Proc. London. Math. Soc. vol. 36 (1933-1934) pp. 451-469.

BROWN UNIVERSITY

Providence, R.I. 